\title{
A mutation analysis of the $A G L$ gene in Korean patients with glycogen storage disease type III
}

\begin{abstract}
Jae Sung Ko ${ }^{1,3}$, Jin Soo Moon ${ }^{1,3}$, Jeong Kee Seo ${ }^{1}$, Hye Ran Yang ${ }^{1}$, Ju Young Chang ${ }^{1}$ and Sung Sup Park ${ }^{2}$
Glycogen storage disease type III (GSD III) is an autosomal recessive disorder that is characterized by the excessive accumulation of abnormal glycogen in the liver and muscles and is caused by a deficiency in glycogen debranching enzyme (amylo-1,6-glucosidase, 4-alpha-glucanotransferase (AGL)) activity. To investigate the molecular characteristics of GSD III patients in Korea, we have sequenced the AGL gene in eight children with GSD III. All patients were compound heterozygotes. We identified 10 different mutations (five novel and five previously reported). The novel mutations include one nonsense (c.1461G > A, p.W487X), three splicing (c.293 +4_293 + 6delAGT in IVS4, c.460+1G > T in IVS5, c.2682-8A $>$ G in IVS21) and one missense mutation (c.2591G >C, p.R864P). Together, p.R285X, c.1735 + 1G > T and p.L1139P accounted for 56\% of all alleles, while the remaining mutations are heterogeneous. These three mutations can be common in Korea, and further larger studies are needed to confirm our findings.
\end{abstract}

Journal of Human Genetics (2014) 59, 42-45; doi:10.1038/jhg.2013.117; published online 21 November 2013

Keywords: $A G L$; glycogen storage disease type III; Korea; mutation

\section{INTRODUCTION}

Glycogen storage disease type III (GSD III) is an autosomal recessive disease that primarily affects the liver, heart and skeletal muscle. It is caused by a deficiency in the activity of the glycogen debranching enzyme amylo-1,6-glucosidase, 4-alpha-glucanotransferase (AGL), which is a key enzyme in the glycogen degradation pathway. ${ }^{1}$ The typical clinical manifestations of GSD III are fasting hypoglycemia, short stature, hepatomegaly, hyperlipidemia and sometimes progressive myopathy and cardiomyopathy. Although hepatic fibrosis is common in children with GSD III, fasting hypoglycemia and hepatomegaly usually improve with age. The majority of GSD III patients display AGL deficiency in both the liver and muscle (subtype IIIa), while only the liver is involved in approximately $15 \%$ of the affected patients (subtype IIIb). ${ }^{2}$

The human AGL gene spans $85 \mathrm{~kb}$ of DNA on chromosome $1 \mathrm{p} 21.2$ and is composed of 35 exons that make up a 7.4-kb mRNA. ${ }^{3}$ To date, at least 110 different $A G L$ gene mutations have been reported in GSD III patients. Mutations in exon 3 have been exclusively associated with the GSD IIIb subtype. ${ }^{2}$ Genetic analyses provide a noninvasive method for diagnosis and GSD III subtype assignment. ${ }^{4}$ Previous studies have revealed that $A G L$ mutation spectra are ethnicityspecific, ${ }^{2,5-8}$ but some mutations are found in different ethnic groups. ${ }^{9-10}$ Currently, the spectrum of mutations in Korean GSD III patients is not well characterized. ${ }^{10-11}$

The aim of this study is to investigate the mutation spectrum of AGL in Korean children with GSD III.

\section{MATERIALS AND METHODS}

\section{Patients}

Eight Korean GSD III children (seven boys and one girl) from eight unrelated families were investigated. The median age was 9.5 years (range: $3-17$ years). GSD III diagnosis was based on a demonstration of excessive glycogen accumulation in a liver biopsy specimen and the identification of pathogenic mutations in both the alleles of the AGL gene. We reviewed the patients' clinical features, including age at the time of diagnosis, growth status and muscle and heart involvement. Laboratory tests, including serum transaminase, bilirubin, albumin, glucose, lipid profiles, uric acid and creatinine kinase (CK) measurements, were performed every 4-6 months. Abdominal ultrasound, electrocardiography and echocardiography were also performed. Those with left ventricular mass above the 95th percentile for height $(z$ score $>1.64)$ by echocardiography were classified as having left ventricular hypertrophy (http://parameterz.blogspot.kr/2008/09/lv-mass-z-scores.html). This study was approved by the institutional review board of the Seoul National University Hospital, Seoul, Korea.

DNA sequencing and mutation analysis of the $A G L$ gene Informed consent was obtained from all of the patients' parents, and patient confidentiality has been maintained. Genomic DNA was extracted from peripheral blood leukocytes using the Wizard genomic DNA purification kit according to the manufacturer's instructions (Promega, Madison, WI, USA). All coding exons and flanking introns of the AGL gene were amplified using PCR. PCR was performed using a thermal cycler (Applied Biosystems, Foster City, CA, USA). PCR products were purified, and direct sequencing was performed on an ABI3100 Genetic Analyzer (Applied Biosystems). The sequences obtained were compared with the reference sequence NG_012865.1

${ }^{1}$ Department of Pediatrics, Seoul National University College of Medicine, Seoul, Korea and ${ }^{2}$ Department of Laboratory Medicine, Seoul National University College of Medicine, Seoul, Korea

${ }^{3}$ These authors contributed equally to this work.

Correspondence: Professor JK Seo, Department of Pediatrics, Seoul National University Hospital, 101 Daehakro, Jongro-gu, Seoul 110-744, Korea.

E-mail: jkseo@snu.ac.kr

Received 26 June 2013; revised 20 September 2013; accepted 27 September 2013; published online 21 November 2013 
registered in the National Center for Biotechnology Information database (http://www.ncbi.nlm.nih.gov). The pathogenicity of a novel missense mutation was investigated by determining the level of conservation of the altered amino acid among various species. PolyPhen (http://genetics.bwh.harvard.edu/ pph2) and SIFT programs (http://sift.jcvi.org) were used to predict the effects of specific amino-acid changes.

\section{RESULTS}

All children initially presented with hepatomegaly with elevated transaminase levels. The median age at the time of diagnosis was 21 months (range 10 months to 3 years) (Table 1). Hypoglycemia was found in 6 children. Abdominal ultrasound showed increased hepatic echogenicity in all the children. Two children had mild muscular weakness when climbing stairs. CK levels were increased in six children. Case 8 who complained of exercise-induced myalgia had very high CK levels. A muscle biopsy of case 6 revealed moderate size variation of myofibers and PAS-positive glycogen accumulation. Echocardiography revealed left ventricular hypertrophy in three children, but they were asymptomatic. One patient exhibited short stature. Liver biopsy demonstrated periportal or septal fibrosis in five patients. None of the patients developed liver cirrhosis.

All patients had compound heterozygous mutations. We identified 10 different AGL mutations, 5 of which are novel: 1 nonsense (c.1461G > A, p.W487X in exon 13), 3 splicing (c.293+4_293+6 delAGT in IVS4, c.460 + $1 \mathrm{G}>\mathrm{T}$ in IVS5, c.2682-8A $>\mathrm{G}$ in IVS21), and 1 missense mutation (c.2591G $>$ C, p.R864P in exon 21). The $G$ to A transition at nucleotide 1461 (c.1461G $>$ A) results in the substitution of the tryptophan residue by a stop codon at codon 487. The deletion of the ATG between c. $293+4$ and $293+6$ in IVS4 causes aberrant splicing and the skipping of exon 4 . The c. $460+1 \mathrm{G}>\mathrm{T}$ mutation in IVS5 is predicted to impair normal splicing, resulting in the skipping of exon 5. The c.2682-8A $>\mathrm{G}$ mutation in IVS21 causes aberrant splicing, resulting in a 7-bp insertion between exons 21 and 22. This insertion produces a frameshift, leading to premature termination at codon 896 (p.L895SfsX2). The $\mathrm{G}$ to $\mathrm{C}$ transition at nucleotide 2591 (c.2591G > C) induces an amino-acid change from an arginine to a proline at codon 864 . The p.R864P mutation is not found in the
NCBI SNP database (http://www.ncbi.nlm.nih.gov/snp). An alignment of AGL amino-acid sequences from various species shows that the arginine at codon 864 is highly conserved through evolution (Figure 1). The PolyPhen program predicted that p.R864P is probably damaging, and the SIFT program predicted that this mutation deleteriously affects protein function.

The previously reported mutations detected here included two nonsense (p.R285X, p.R977X), one splicing (c.1735+1G>T) and two missense mutations (p.R675W, p.L1139P). Three common mutations were identified. The allele frequencies of p.R285X, c.1735 $+1 \mathrm{G}>\mathrm{T}$ and p.L1139P were $18.8 \%$ each. Thus, these three common mutations account for $56 \%$ of all mutant alleles.

\section{DISCUSSION}

Our molecular analysis of 8 Korean GSD III patients revealed 10 different mutations (5 novel and 5 previously reported). A novel nonsense mutation ( $\mathrm{p} . \mathrm{W} 487 \mathrm{X}$ ) produced a premature termination codon. Three novel splicing mutations (c.293+4_293+6delAGT, c. $460+1 \mathrm{G}>\mathrm{T}, \mathrm{c} .2682-8 \mathrm{~A}>\mathrm{G}$ ) were expected to result in premature termination. Most of the reported mutations in the AGL gene are expected to result in premature stop codons. ${ }^{12}$ A novel missense mutation (p.R864P) was also identified. The arginine that is altered by this mutation is strongly evolutionarily conserved, and another mutation in the same codon (p.R864X) has been reported previously. ${ }^{2}$

Most AGL mutations have been reported in only one or a few patients. Mutations in GSD IIIa are scattered throughout the AGL gene and exhibit allelic heterogeneity. ${ }^{12-14}$ Unlike GSD IIIa, GSD IIIb is associated with two specific mutations in exon 3, c.18_19delGA (p.Q6HfsX20) and c.16C > T (p.Q6X). ${ }^{2}$

We have shown that all patients had AGL mutations located outside of exon 3. Although two children showed myopathic symptoms and six children had elevated CK levels, a molecular diagnosis of GSD IIIa was made in all the patients. Muscular involvement in GSD IIIa is variable, and a normal CK value does not rule out muscle involvement. Muscle weakness is usually minimal during childhood, but progressive myopathy can develop after the third or fourth decade of life. Although left ventricular hypertrophy is found in three children,

Table 1 Clinical features and genotypes of GSD III patients

\begin{tabular}{|c|c|c|c|c|c|c|c|c|c|}
\hline $\begin{array}{l}\text { Case } \\
\text { no. }\end{array}$ & Age/sex & Age at diagnosis & Clinical findings & Echo & $\begin{array}{c}C K \\
\left(\mid U I^{-1}\right)\end{array}$ & $\begin{array}{c}\text { Height } \\
\text { (percentile) }\end{array}$ & $\begin{array}{c}\text { Weight } \\
\text { (percentile) }\end{array}$ & Liver biopsy & Genotype \\
\hline 1 & 3 years/B & 17 months & Hepatomegaly, hypoglycemia & $\mathrm{NI}$ & 204 & $<3 r d$ & 25-50th & Glycogen, fibrosis & $\begin{array}{l}\text { p.R285X } \\
\text { p.L1139P }\end{array}$ \\
\hline 2 & 5 years/G & 23 months & Hepatomegaly, hypoglycemia & $\mathrm{NI}$ & 63 & 25-50th & 90-95th & Glycogen, fibrosis & $\begin{array}{l}\text { p.R285X } \\
\text { p.R675W }\end{array}$ \\
\hline 3 & 4 years/B & 10 months & Hepatomegaly, hypoglycemia & LVH & 857 & 50th & $50-75$ th & Glycogen, fibrosis & $\begin{array}{l}\text { c. } 460+1 \mathrm{G}>\mathrm{T} \\
\text { c. } 1735+\mathrm{G}>\mathrm{T}\end{array}$ \\
\hline 4 & 7 years/B & 17 months & Hepatomegaly, hypoglycemia & $\mathrm{NI}$ & 507 & $25-50$ th & $50-75$ th & Glycogen, fibrosis & $\begin{array}{l}\text { p.R285X } \\
\text { c. } 293+4 \_293+6 \text { delAGT }\end{array}$ \\
\hline 5 & 12 years/B & 27 months & Hepatomegaly, hypoglycemia & $\mathrm{NI}$ & 91 & $10-25$ th & 10-25th & Glycogen & $\begin{array}{l}\text { c. } 1735+\mathrm{G}>\mathrm{T} \\
\text { p.R864P }\end{array}$ \\
\hline 6 & 12 years/B & 19 months & Hepatomegaly, mild weakness & LVH & 489 & 75-90th & 75-90th & Glycogen & $\begin{array}{l}\text { p.L1139P } \\
\text { c. } 1735+\mathrm{G}>\mathrm{T}\end{array}$ \\
\hline 7 & 15 years/B & 36 months & Hepatomegaly & LVH & 437 & 25-50th & 10-25th & Glycogen & $\begin{array}{l}\text { p.L1139P } \\
\text { p.W487X }\end{array}$ \\
\hline 8 & 17 years/B & 26 months & $\begin{array}{l}\text { Hepatomegaly, hypoglycemia, } \\
\text { myalgia, mild weakness }\end{array}$ & $\mathrm{NI}$ & 5791 & $50-75$ th & 25-50th & Glycogen, fibrosis & $\begin{array}{l}\text { c. } 2682-8 A>G \\
\text { p.R977X }\end{array}$ \\
\hline
\end{tabular}

Abbreviations: B, boy; CK, creatinine kinase; Echo, echocardiography; G, girl; GSD, glycogen storage disease; LVH, left ventricular hypertrophy; NI, normal.

Glycogen means excess accumulation of glycogen in the liver. 


\begin{tabular}{|c|c|c|c|c|c|c|c|c|c|}
\hline Human (H. sapiens), NP_000019 & $\mathrm{G}$ & $\mathrm{I}$ & $\mathrm{L}$ & $\mathrm{R}$ & $\mathrm{N}$ & $\mathrm{H}$ & $\mathrm{L}$ & $\mathrm{T}$ & Q \\
\hline Chimpanzee (P.troglodytes), XP_524777 & $\mathrm{G}$ & 1 & $\mathrm{~L}$ & $\mathrm{R}$ & $\mathrm{N}$ & $\mathrm{H}$ & $\mathrm{L}$ & $\mathrm{T}$ & Q \\
\hline Cow (B. taurus), XP_595566 & $\mathrm{G}$ & I & $\mathrm{L}$ & $\mathrm{R}$ & $\mathrm{N}$ & $\mathrm{H}$ & $\mathrm{L}$ & $\mathrm{T}$ & Q \\
\hline Horse (E. caballus), NP_001103778 & $\mathrm{G}$ & I & $\mathrm{L}$ & $\mathrm{R}$ & $\mathrm{N}$ & $\mathrm{H}$ & $\mathrm{L}$ & $T$ & Q \\
\hline Rabbit (O. cuniculus), NP_001075716 & G & I & $\mathrm{L}$ & $\mathrm{R}$ & $\mathrm{N}$ & $\mathrm{H}$ & $\mathrm{L}$ & $\Gamma$ & 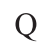 \\
\hline Dog (C. familiaris), NP_001041561 & G & I & $\mathrm{L}$ & $\mathrm{R}$ & $\mathrm{N}$ & $\mathrm{H}$ & $\mathrm{L}$ & $\mathrm{T}$ & Q \\
\hline Mouse (M. musculus), NP_001074795 & $\mathrm{G}$ & I & $\mathrm{L}$ & $\mathrm{R}$ & $\mathrm{N}$ & $\mathrm{H}$ & $\mathrm{L}$ & $\Gamma$ & Q \\
\hline Rat (R. norvegicus), NP_001102034 & $\mathrm{G}$ & I & $\mathrm{L}$ & $\mathrm{R}$ & $\mathrm{N}$ & $\mathrm{H}$ & $\mathrm{L}$ & 1 & Q \\
\hline Chicken (G. gallus), XP_422317 & $\mathrm{G}$ & $\mathrm{V}$ & $\mathrm{L}$ & $\mathrm{R}$ & $\mathrm{N}$ & $\mathrm{H}$ & $\mathrm{L}$ & I & Q \\
\hline Zebra fish (D. rerio), NP_001166124 & G & $\mathrm{V}$ & $\mathrm{L}$ & $1 \mathrm{~T}$ & $\mathrm{~N}$ & $\mathrm{H}$ & $\mathrm{L}$ & I & $\mathrm{Q}$ \\
\hline
\end{tabular}

Figure 1 Amino-acid sequences surrounding position 864 of AGL homologues from different species.

symptomatic cardiomyopathy is rare in GSD III patients. ${ }^{1}$ Liver histology showed periportal or septal fibrosis in five children. Liver disease, however, is usually self-limited, and the major cause of morbidity is muscle disease.

Several prevalent mutations have been reported in ethnic groups with high rates of consanguinity. Because of a founder effect, Faroe islanders have the highest prevalence (1 in 3100) of GSD IIIa worldwide. ${ }^{8}$ Six children with GSD IIIa from the Faroe Islands have been found to be homozygous for p.R408X. Similarly, the c.4455delT in homozygous form was found in 12 North African Jewish patients with GSD III, and a founder mutation explains the high prevalence of GSD III ( 1 in 5400 ) in this population. ${ }^{5}$

Genetic heterogeneity has been observed in Italian patients with GSD III, although c.664 +3A $>$ G (18.2\%) and c.2681+1G $>$ A $(18.2 \%)$ were common mutations. ${ }^{15}$ In the United States, p.R864X (10.3\%), c.3964delT (6.7\%), IVS32-12A > G (5.5\%) and p.R1228X $(5.2 \%)$ are the most frequent mutations, but they account for only $28 \%$ of all mutant alleles. ${ }^{16}$ In Japan, 11 different mutations have been reported. ${ }^{17}$ Genetic heterogeneity has also been reported in Chinese, ${ }^{9}$ Egyptian, ${ }^{18}$ Turkish $^{7}$ and Irish ${ }^{19}$ patients.

We found that in Korean patients with GSD III, three common mutations (p.R285X, c.1735 + 1G > T and p.L1139P) are responsible for $>50 \%$ of the total alleles, while the remaining mutations are heterogeneous. These three common mutations have been reported previously in Korean patients. ${ }^{10-11}$ The c.1735 + 1G $>\mathrm{T}$ in IVS14, which has been identified in two Korean patients and one Japanese patient, causes the skipping of exon 14, leading to a truncated enzyme. ${ }^{10,20}$ The p.R285X mutation in exon 8 has also been reported in Japanese and Italian patients. ${ }^{15,21}$ The p.L1139P mutation, previously identified in a Korean patient, ${ }^{11}$ is located in exon 27 , which is known to belong to the glucosidase domain. The p.R977X in exon 23 has been previously reported in Italian patients. ${ }^{15} \mathrm{~A}$ p.R675W mutation in exon 17 has been identified in a Tunisian patient. $^{22}$ These results suggest that p.P285X, p.R977X and p.R675W are recurrent mutations in various ethnic groups. To date, a total of 15 different mutations, including 5 (p.R428K, c.1306delA, c.1510_1511insT, c.2894_2896delGGAinsTG and p.D1364H) that were not found in this study, ${ }^{10-11}$ have been identified in Korean patients with GSD III. The p.R285X, c.1735 + 1G > T and p.L1139P alleles may be useful for genetic screening in Korea, but our findings also confirm the genetic heterogeneity of GSD III in the Korean population.
No clear genotype-phenotype correlation has been elucidated for GSD IIIa. Because all patients in this study are compound heterozygotes, it is difficult to investigate genotype-phenotype correlations. Previous studies suggest that homozygous mutations in exons 28, 31 and 32 are associated with severe phenotypes in patients with GSD III. ${ }^{19,23-24}$ It has been suggested that exons $31-34$ are functionally significant, because they encode the glycogen-binding domain of AGL. ${ }^{14}$ However, the severity of clinical features varies even in siblings who have the same genotype. ${ }^{6,24}$

In summary, we identified 10 different mutations in 8 Korean GSD III patients. Of these 10 mutations, 5 are novel: p.W487X, c.293+ 4_293+6delAGT, c.460+1G>T, c.2682-8A > G, and p.R864P. Together, p.R285X, c.1735 $+1 \mathrm{G}>\mathrm{T}$ and p.L1139P accounted for $56 \%$ of all alleles, and the remaining mutations are heterogeneous. These three mutations can be common in Korea, and further larger genetic studies are needed to confirm our findings.

\section{CONFLICT OF INTEREST}

The authors declare no conflict of interest.

\section{ACKNOWLEDGEMENTS}

This study was supported by Grant no. 0420120490 from the SNUH Research Fund.

1 Coleman, R. A., Winter, H. S., Wolf, B., Gilchrist, J. M. \& Chen, Y. T. Glycogen storage disease type III (glycogen debranching enzyme deficiency): correlation of biochemical defects with myopathy and cardiomyopathy. Ann. Intern. Med. 116, 896-900 (1992).

2 Shen, J., Bao, Y., Liu, H. M., Lee, P., Leonard, J. V. \& Chen, Y. T. Mutations in exon 3 of the glycogen debranching enzyme gene are associated with glycogen storage disease type III that is differentially expressed in liver and muscle. J. Clin. Invest. 98, 352-357 (1996).

3 Bao, Y., Dawson, T. L. Jr. \& Chen, Y. T. Human glycogen debranching enzyme gene (AGL): complete structural organization and characterization of the $5^{\prime}$ flanking region. Genomics 38, 155-165 (1996).

4 Kishnani, P. S., Austin, S. L., Arn, P., Bali, D. S., Boney, A., Case, L. E. et al Glycogen storage disease type III diagnosis and management guidelines. Genet. Med. 12, 446-463 (2010).

5 Parvari, R., Moses, S., Shen, J., Hershkovitz, E., Lerner, A. \& Chen, Y. T. A single-base deletion in the $3^{\prime}$-coding region of glycogen-debranching enzyme is prevalent in glycogen storage disease type IIIA in a population of North African Jewish patients. Eur. J. Hum. Genet. 5, 266-270 (1997).

6 Mili, A., Ben Charfeddine, I., Mamai, O., Abdelhak, S., Adala, L., Amara, A. et al. Molecular and biochemical characterization of Tunisian patients with glycogen storage disease type III. J. Hum. Genet. 57, 170-175 (2012). 
7 Aoyama, Y., Ozer, I., Demirkol, M., Ebara, T., Murase, T., Podskarbi, T. et al. Molecular features of 23 patients with glycogen storage disease type III in Turkey: a nove mutation p.R1147G associated with isolated glucosidase deficiency, along with 9 AGL mutations. J. Hum. Genet. 54, 681-686 (2009).

8 Santer, R., Kinner, M., Steuerwald, U., Kjaergaard, S., Skovby, F., Simonsen, H. et al. Molecular genetic basis and prevalence of glycogen storage disease type IIIA in the Faroe Islands. Eur. J. Hum. Genet. 9, 388-391 (2001).

9 Lam, C. W., Lee, A. T., Lam, Y. Y., Wong, T. W., Mak, T. W., Fung, W. C. et al. DNA-based subtyping of glycogen storage disease type III: mutation and haplotype analysis of the AGL gene in Chinese. Mol. Genet. Metab. 83, 271-275 (2004).

10 Oh, S. H., Park, H. D., Ki, C. S., Choe, Y. H. \& Lee, S. Y. Biochemical and molecular investigation of two Korean patients with glycogen storage disease type III. Clin. Chem. Lab. Med. 46, 1245-1249 (2008).

11 Ko, J. M., Kim, G. H. \& Yoo, H. W. AGL gene mutation and clinical features in Korean patients with glycogen storage disease type III. J. Genet. Med. 4, 72-79 (2007).

12 Goldstein, J. L., Austin, S. L., Boyette, K., Kanaly, A., Veerapandiyan, A., Rehder, C. et al. Molecular analysis of the AGL gene: identification of 25 novel mutations and evidence of genetic heterogeneity in patients with Glycogen Storage Disease Type III. Genet. Med. 12, 424-430 (2010).

13 Okubo, M., Horinishi, A., Takeuchi, M., Suzuki, Y., Sakura, N., Hasegawa, Y. et al. Heterogeneous mutations in the glycogen-debranching enzyme gene are responsible for glycogen storage disease type IIla in Japan. Hum. Genet. 106, 108-115 (2000).

14 Endo, Y., Horinishi, A., Vorgerd, M., Aoyama, Y., Ebara, T., Murase, T. et al. Molecular analysis of the AGL gene: heterogeneity of mutations in patients with glycogen storage disease type III from Germany, Canada, Afghanistan, Iran, and Turkey. J. Hum. Genet. 51, 958-963 (2006).
15 Lucchiari, S., Pagliarani, S., Salani, S., Filocamo, M., Di Rocco, M., Melis, D. et al. Hepatic and neuromuscular forms of glycogenosis type III: nine mutations in AGL. Hum. Mutat. 27, 600-601 (2006).

16 Shen, J. J. \& Chen, Y. T. Molecular characterization of glycogen storage disease type III. Curr. Mol. Med. 2, 167-175 (2002).

17 Horinishi, A., Okubo, M., Tang, N. L., Hui, J., To, K. F., Mabuchi, T. et al. Mutational and haplotype analysis of AGL in patients with glycogen storage disease type III. J. Hum. Genet. 47, 55-59 (2002).

18 Endo, Y., Fateen, E., Aoyama, Y., Horinishi, A., Ebara, T., Murase, T. et al. Molecular characterization of Egyptian patients with glycogen storage disease type IIIa. J. Hum. Genet. 50, 538-542 (2005).

19 Crushell, E., Treacy, E. P., Dawe, J., Durkie, M. \& Beauchamp, N. J. Glycogen storage disease type III in the Irish population. J. Inherit. Metab. Dis. 3, 215-218 (2010).

20 Okubo, M., Aoyama, Y. \& Murase, T. A novel donor splice site mutation in the glycogen debranching enzyme gene is associated with glycogen storage disease type III. Biochem. Biophys. Res. Commun. 225, 695 (1996).

21 Ogimoto, A., Okubo, M., Okayama, H., Shin, Y. S., Endo, Y., Ebara, T. et al. A Japanese patient with cardiomyopathy caused by a novel mutation R285X in the AGL gene. Circ. J. 71, 1653-1656 (2007).

22 Lucchiari, S., Donati, M. A., Parini, R., Melis, D., Gatti, R., Bresolin, N. et al. Molecular characterisation of GSD III subjects and identification of six novel mutations in AGL. Hum. Mutat. 20, 480 (2002).

23 Shaiu, W. L., Kishnani, P. S., Shen, J., Liu, H. M. \& Chen, Y. T. Genotype-phenotype correlation in two frequent mutations and mutation update in type III glycogen storage disease. Mol. Genet. Metab. 69, 16-23 (2000).

24 Lucchiari, S., Fogh, I., Prelle, A., Parini, R., Bresolin, N., Melis, D. et al. Clinical and genetic variability of glycogen storage disease type IIla: seven novel AGL gene mutations in the Mediterranean area. Am. J. Med. Genet. 109, 183-190 (2002). 